OPEN ACCESS

Edited by:

Antonio Faciola,

University of Florida, United States

Reviewed by:

Brooke Ashley Clemmons, Texas A\&M University-Commerce,

United States

Fernanda Rosa,

University of Arkansas for Medical

Sciences, United States

*Correspondence:

Yangdong Zhang

zhangyangdong@caas.cn Jiaqi Wang

jiagiwang@vip.163.com

Specialty section:

This article was submitted to Systems Microbiology,

a section of the journal

Frontiers in Microbiology

Received: 18 August 2021 Accepted: 18 October 2021 Published: 12 November 2021

Citation:

Huang G, Guo L, Chang X, Liu K, Tang $W$, Zheng $N$, Zhao S, Zhang $Y$ and Wang J (2021) Effect of Whole or Ground Flaxseed Supplementation on

Fatty Acid Profile, Fermentation, and Bacterial Composition in Rumen

of Dairy Cows.

Front. Microbiol. 12:760528. doi: 10.3389/fmicb.2021.760528

\section{Effect of Whole or Ground Flaxseed Supplementation on Fatty Acid Profile, Fermentation, and Bacterial Composition in Rumen of Dairy Cows}

\author{
Guoxin Huang 1,2,3, Liya Guo ${ }^{4}$, Xiaofeng Chang ${ }^{1}$, Kaizhen Liu' ${ }^{2,3}$, Wenhao Tang ${ }^{2,3}$, \\ Nan Zheng ${ }^{2,3}$, Shengguo Zhao ${ }^{2,3}$, Yangdong Zhang ${ }^{2,3 *}$ and Jiaqi Wang ${ }^{1,2,3 *}$ \\ ${ }^{1}$ College of Animal Sciences and Technology, Northeast Agricultural University, Harbin, China, ${ }^{2}$ Key Laboratory of Quality \& \\ Safety Control for Milk and Dairy Products of Ministry of Agriculture and Rural Affairs, Institute of Animal Sciences, Chinese \\ Academy of Agricultural Sciences, Beijing, China, ${ }^{3}$ State Key Laboratory of Animal Nutrition, Institute of Animal Sciences, \\ Chinese Academy of Agricultural Sciences, Beijing, China, ${ }^{4}$ Henan Institute of Science and Technology, Xinxiang, China
}

Flaxseed is rich in $\alpha$-linolenic acid (ALA) and can increase omega-3 polyunsaturated fatty acid in the milk of dairy cows. However, the response of rumen fermentation to different forms of flaxseed supplementation is unknown. This study aimed to investigate the effect of different forms of flaxseed on the fatty acid profile, fermentation, and composition of bacteria in the rumen of dairy cows. In total, 30 Holstein dairy cows were selected and randomly assigned into three groups (10/group). Cows were fed a basal diet (control check; CK) or basal diets supplemented with either 1,500 g per day whole flaxseed (WF) or 1,500 g per day ground flaxseed (GF). The WF group had the highest ALA content in rumen fluid, whereas no difference was found between the CK and GF groups. However, the molar proportion of acetate increased in the WF and GF groups and was the highest in the GF group, and a similar trend was shown by propionate, isobutyrate, butyrate, isovalerate, and valerate (CK $<\mathrm{WF}<\mathrm{GF}$ ). The abundance of Ruminococcaceae_NK4A214_group, Christensenellaceae_R7_group, and Eubacterium_coprostanoligenes_group also showed the same trend (CK $<$ WF $<$ GF). Different forms of flaxseed release ALA by different mechanisms in the rumen, and the molar proportions of volatile fatty acids and the bacterial composition were potentially influenced mainly by the amount of ALA released into the rumen.

Keywords: flaxseed, $\alpha$-linolenic acid, rumen fatty acid, rumen metabolism, bacteria

\section{INTRODUCTION}

As a source of omega-3 polyunsaturated fatty acid (n-3 PUFA), flaxseed is widely used to enhance levels of n-3 PUFA in milk production (Meignan et al., 2017; Brzozowska et al., 2018; Marino et al., 2019), particularly, $\alpha$-linolenic acid (ALA; c9,c12,c15-C18:3). Flaxseed is rich in ALA, 18\% of the total seed, and 53 to $56 \%$ of total fatty acids (Mustafa et al., 2002; Petit, 2003). In feeding experiments, whole (WF; Castillo-Lopez et al., 2018) and ground flaxseed (GF; Isenberg et al., 2019) are two important forms of dietary supplementation. However, previous studies reported that GF supplementation had a higher efficiency in increasing n-3 PUFA (mainly ALA) in milk 
(Silva et al., 2007; Petit and Cortes, 2010; Mesgaran et al., 2012). The n-3 PUFA (mainly ALA) in WF cannot be released as completely as GF because of the flaxseed shell. Most ALA in WF is excreted directly from the animal (Oba et al., 2009). To release ALA from WF, cows need to crush the flaxseed by tooth. Thus, in the rumen of ruminants, ALA in WF takes longer to be released. However, ALA in GF could be released into the rumen in a shorter time. Compared to a short and rapid release, a long release may lead to the continuous biological hydrogenation of ALA in the rumen. Therefore, the efficiency of transporting the ALA into milk is lower.

In the rumen, dietary PUFA can influence the composition of the microbiota (Fievez et al., 2007). Previous articles have reported that ALA was toxic to the growth of Clostridium proteoclasticum, Butyrivibrio hungatei, and Eubacterium ruminantium in the rumen (Maia et al., 2007). The release of ALA from either WF or GF is different and may influence the ruminal microbiota diversity. However, no studies have revealed the influence of ALA from different flaxseed on the microflora ruminal.

This study aimed to investigate the effect of WF or GF supplementation on rumen formation and bacterial community. We hypothesized that dietary supplementation with WF and GF may have a different effect on the rumen fatty acid profile and that the rumen fermentation pattern and the structure of bacteria may be influenced by the ALA released into the rumen.

\section{MATERIALS AND METHODS}

\section{Experimental Design and Treatments}

This experiment was performed at Tianjin Fuyou Agricultural Technology Co., Ltd. (Tianjin, China). The animal protocol (protocol no. IAS 2019-28; date of approval: April 8, 2019) was approved by the Animal Care and Use Committee of the Institute of Animal Science, Chinese Academy of Agricultural Sciences, Beijing, China. Thirty primiparous Holstein dairy cows (90 \pm 28 days in milk; $628 \pm 103 \mathrm{~kg}$ body weight; $37.22 \pm 2.60 \mathrm{~kg}$ of milk per day) were selected for the present study. Cows were randomly assigned into three groups and provided with a basal diet (control check; CK) or the basal diet supplemented with 1,500 g per day WF or 1,500 g per day GF, as shown in Table 1 (predicted feed intake: $22.3 \mathrm{~kg}$ per day and dry matter of flaxseed is $94.96 \%$, thus flaxseed content in diet: $6.38 \%$ ). The diets in this experiment were formulated and evaluated using the Feeding Standards of Dairy Cattle in China (Ministry of Agriculture of China (MOA). Feeding Standard of Dairy Cattle, NY/T 34-2004; MOA: Beijing, China, 2004). This experiment lasted for 5 weeks, and the cows were fed in a well-ventilated barn. The daily feeding times were 6:30 AM, 2:00 PM, and 9:30 PM using a total mixed ratio. In this experiment, the dry matter intake and milk yield showed no difference between the treatments.

\section{Sampling, Measurements, and Analysis}

The rumen fluid was collected at 6:00 AM via esophageal tubing, which was described by Paz et al. (2016). Approximately, $100 \mathrm{~mL}$ of rumen fluid was collected and sieved through four
TABLE 1 | Composition of experimental diets (\% DM basis).

\begin{tabular}{|c|c|c|c|}
\hline \multirow[t]{2}{*}{ Items } & \multicolumn{3}{|c|}{ Treatments $^{a}$} \\
\hline & CK & WF & GF \\
\hline \multicolumn{4}{|l|}{ Diet ingredient, \% of DM } \\
\hline Corn silage & 21.51 & 21.86 & 21.86 \\
\hline Alfalfa hay & 8.76 & 8.90 & 8.90 \\
\hline Alfalfa semidry silage & 3.61 & 3.67 & 3.67 \\
\hline Wrapped straw & 2.44 & 2.48 & 2.48 \\
\hline Corn & 26.18 & 21.56 & 21.56 \\
\hline Corn flakes & 2.77 & 2.82 & 2.82 \\
\hline Wool cotton seed & 3.88 & 1.97 & 1.97 \\
\hline Orange peel granule & 1.94 & 1.97 & 1.97 \\
\hline Soybean meal & 12.42 & 11.8 & 11.8 \\
\hline Puffed soybeans & 2.22 & 2.26 & 2.26 \\
\hline Cotton meal & 3.12 & 3.17 & 3.17 \\
\hline DDGS & 2.85 & 2.90 & 2.90 \\
\hline Bran & 1.08 & 1.10 & 1.10 \\
\hline Fat powder & 1.71 & 1.55 & 1.55 \\
\hline Flaxseed & 0 & 6.38 & 6.38 \\
\hline Premix ${ }^{b}$ & 5.51 & 5.60 & 5.60 \\
\hline Total & 100 & 100 & 100 \\
\hline \multicolumn{4}{|l|}{ Chemical, \% of DM } \\
\hline DM & 51.18 & 51.27 & 53.58 \\
\hline $\mathrm{NE}_{\mathrm{L}}{ }^{\mathrm{c}}, \mathrm{Mcal} / \mathrm{kg}$ of $\mathrm{DM}$ & 1.91 & 1.94 & 1.94 \\
\hline $\mathrm{CP}$ & 17.19 & 17.48 & 17.48 \\
\hline ADF & 17.99 & 19.30 & 17.08 \\
\hline NDF & 63.15 & 65.70 & 61.23 \\
\hline EE & 4.59 & 8.52 & 8.50 \\
\hline \multicolumn{4}{|c|}{ FA, \% of total FA reported } \\
\hline C14:0 & 2.04 & 1.43 & 1.46 \\
\hline C16:0 & 76.05 & 67.02 & 68.04 \\
\hline c9-C16:1 & 0.03 & 0.03 & 0.04 \\
\hline C18:0 & 0.03 & 0.04 & 0.05 \\
\hline c9-C18:1 & 0.77 & 0.94 & 1.01 \\
\hline c9,c12-C18:2 & 18.86 & 9.84 & 9.49 \\
\hline c9,c12,c15-C18:3 & 2.10 & 20.60 & 19.80 \\
\hline C20:0 & 0.04 & 0.03 & 0.04 \\
\hline c11-C20:1 & 0.01 & 0.01 & 0.01 \\
\hline C22:0 & 0.04 & 0.03 & 0.04 \\
\hline C24:0 & 0.03 & 0.02 & 0.03 \\
\hline
\end{tabular}

DM, dry matter; NEL, net energy for lactation; CP, crude protein; ADF, acid detergent fiber; NDF, neutral detergent fiber; EE, ether extracts.

${ }^{a}$ CK, dairy cows fed a basal diet (without flaxseed); WF, dairy cows fed whole flaxseed diet (whole flaxseed 1,500 g per day); GF, dairy cows fed with ground flaxseed diet (ground flaxseed 1,500 g per day per cow).

bPremix (per kg of DM): a minimum of $313,500 \mathrm{U}$ of vitamin A, 104,500 $\mathrm{U}$ of vitamin D, 5,000 U of vitamin E, $780 \mathrm{mg}$ of $\mathrm{Cu}, 780 \mathrm{mg}$ of $\mathrm{Fe}, 780 \mathrm{mg}$ of $\mathrm{Mn}$, $3,900 \mathrm{mg}$ of $\mathrm{Zn}, 30 \mathrm{mg}$ of Se, $50 \mathrm{mg}$ of I, and $65 \mathrm{mg}$ of Co.

${ }^{c}$ Calculated value (based on China Standard NY/T 34; People's Republic of China, 2004).

cheesecloth layers. The first $10 \mathrm{~mL}$ was used to analyze the $\mathrm{pH}$ value using a $\mathrm{pH}$ meter (UB-10 $\mathrm{pH}$ meter, United States); the remaining rumen fluid was placed into eight $5-\mathrm{mL}$ plastic cryotubes and stored in liquid nitrogen for further analysis. The rumen ammonia-N $\left(\mathrm{NH}_{3}-\mathrm{N}\right)$ concentration was determined 
according to the method by Feng and Gao (1993) and volatile fatty acid (VFA) concentration by using gas chromatography (Agilent Technologies, Santa Clara, CA, United States) (Hu et al., 2005). Ruminal fatty acid analysis was according to the method described by Dreiucker and Vetter (2011).

\section{DNA Extraction}

The total rumen bacteria DNA was extracted by using cetyltrimethylammonium bromide (CTAB). Rumen fluid $(1.5 \mathrm{~mL})$ was centrifuged at $13,000 \times g$ at $4^{\circ} \mathrm{C}$ for $10 \mathrm{~min}$ to collect the supernatant, which contains rumen bacteria. The supernatant and $800 \mu \mathrm{L}$ CTAB (100 mM Tris- $\mathrm{HCl}, \mathrm{pH}$ 8.0; 1.4 M NaCl; 20 mM EDTA; 2\% CTAB) were mixed together and crushed by using a Mixer Mill MM 400 (Retsch, Haan, Germany) at a vibrational frequency of $30 \mathrm{~m} / \mathrm{s}$ for $1 \mathrm{~min}$. Next, the mixtures were incubated at $70^{\circ} \mathrm{C}$ for $20 \mathrm{~min}$, and the supernatant was separated by centrifuge $(13,000 \times g$ for $10 \mathrm{~min})$. Then, $700 \mu \mathrm{L}$ of supernatant was mixed with $600 \mathrm{~mL}$ of phenolchloroformisoamyl alcohol (volume 25:24:1), and $500 \mu \mathrm{L}$ of the upper liquid was transferred into a new tube and mixed with 0.8 times the volume of isopropanol and centrifuged at $13,000 \times g$ for $10 \mathrm{~min}$ at $4^{\circ} \mathrm{C}$. The supernatant was removed from the tube and placed into $800 \mu \mathrm{L}$ ethanol (70\%) to precipitate the DNA. The quality of extracted DNA was evaluated through agarose gel electrophoresis and Nanodrop spectrometer (Thermo Scientific, Chicago, IL, United States). The average concentration of DNA was $396 \mathrm{ng} / \mu \mathrm{L}$ and the average of the $260 / 280$ ratio was 1.88 for all samples.

\section{High-Throughput Sequencing of the 16S rDNA Gene}

The V3 and V4 regions of $16 \mathrm{~S}$ rDNA were selected for amplification by polymerase chain reaction (PCR) with the universal primers $341 \mathrm{~F}$ ( $5^{\prime}$-CCTACGGGNGGCWGCAG) and 806R (5'-GGACTACHVGGGTATCTAAT) (Wang C. et al., 2019). The PCR incubation conditions were performed with KOD Polymerase (Toyobo, Osaka, Japan) at $95^{\circ} \mathrm{C}$ for $4 \mathrm{~min}$, followed by 15 cycles at $95^{\circ} \mathrm{C}$ for $60 \mathrm{~s}, 60^{\circ} \mathrm{C}$ for $40 \mathrm{~s}$, and $72^{\circ} \mathrm{C}$ for $60 \mathrm{~s}$ and a final extension at $72^{\circ} \mathrm{C}$ for $10 \mathrm{~min}$. The PCR products were collected using an AxyPrep DNA Gel Extraction Kit (Axygen Biosciences, Union City, CA, United States).

\section{Sequencing Data Processing}

The 16S rDNA amplicon sequencing was sequenced on an Illumina Novaseq 6000 platform (Guangdong Magigene Biotechnology, Guangzhou, China). QIIME (Quantitative Insights Into Microbial Ecology, version 1.9.1) was used in this experiment to analyze the sequences. According to the overlap relationship between pair-end reads, the sequence data were merged into one sequence tag. After that, UCLUST was used to cluster tags according to $97 \%$ similarity level. Naive Bayesian assignment algorithm of RDP Classifier (version 2.2) was used to identify those representative sequences against the Greengene database (version gg_13_5). $\alpha$-Diversity (Ace, Chao, Good coverage, Shannon, Simpson, Sobs) and $\beta$-diversity PCoA (principal coordinate analysis) and ANOSIM were performed using QIIME (version 1.9.1). The sequence data have been submitted to the Sequence Read Archive of the NCBI under project PRJNA756791.

\section{Statistical Analysis}

Homogeneity of variance and normal distribution for the variables of $\mathrm{pH}, \mathrm{NH}_{3}-\mathrm{N}, \mathrm{VFAs}$, and fatty acid composition of the rumen were tested by the HOVTEST and UNIVARIATE of the SAS (version 9.4, SAS Institute, Inc., Cary, NC, United States) software. Then the data were analyzed using one-way analysis of variance models in SAS. The following statistical model was used:

$$
Y_{\mathrm{ij}}=\mu+T_{\mathrm{i}}+\varepsilon_{\mathrm{ij}},
$$

where $Y_{\mathrm{ij}}$ represents the observed dependent variables, $\mu$ is the overall mean, $T_{\mathrm{i}}$ is the effect of treatment, and $\varepsilon_{\mathrm{ij}}$ is the residual error. The significance level was declared at $P<0.05$.

\section{RESULTS}

\section{Rumen Fatty Acid Profiles}

The concentration of fatty acid in rumen fluid is shown in Table 2. The proportion of C6:0 (caproic), C8:0 (caprylic), C10:0 (capric), C12:0 (lauric), and C14:0 (myristic) was no different between treatments. However, supplementation with GF increased the concentration of C16:0 (palmitic) in the rumen, compared with the WF and CK groups. The concentration of C18:1-trans (elaidic acid) in rumens decreased in WF compared with the $\mathrm{CK}$ and GF groups $(P=0.018)$. However, the levels of ALA $(P=0.039)$ and total $n-3$ PUFA $(P=0.036)$ were greater in the WF group relative to $\mathrm{CK}$ and GF groups. Supplementation with WF tended to increase the concentration of EPA compared with the $\mathrm{CK}$ and GF groups, but there was no significant difference between them $(P=0.099)$.

\section{Ruminal Fermentation Parameters}

The indices of ruminal fermentation are shown in Table 3. Flaxseed supplementation had no influence on the rumen $\mathrm{pH}$ $(P=0.115)$, and no differences were found in ruminal $\mathrm{NH}_{3}-\mathrm{N}$ between the three groups $(P=0.630)$. The molar proportion of VFAs showed differences between the treatments. Compared to the CK group, the molar proportion of acetate increased in the WF and GF groups and was highest in the GF group $(P<0.001)$. In addition, propionate, isobutyrate, butyrate, isovalerate, and valerate showed the same trend $\mathrm{CK}<\mathrm{WF}<\mathrm{GF}(P<0.05)$. Ground and WF supplementation could decrease the ratio of acetate to propionate $(P<0.001)$.

\section{Sequencing and $\alpha$-Diversity Measures}

A total of 3,134,077 high-quality sequences were generated in the partial 16S rRNA gene amplicon sequencing and an average of $103,622 \pm 7,687$ in the rumen. $\alpha$-Diversity measures are shown in Table 4. All the indexes of $\alpha$-diversity measures were not significantly different between the CK and WF groups $(P<0.05)$. However, compared with the CK and WF group, the Shannon $(P<0.001)$, Simpson $(P=0.004)$, and Ace $(P=0.039)$ indexes 
TABLE 2 | Effects of dietary whole or ground flaxseed supplementation on rumen fatty acid ( $\mathrm{g} / 100 \mathrm{~g}$ total fatty acid).

\begin{tabular}{|c|c|c|c|c|}
\hline \multirow[t]{2}{*}{ Items } & \multicolumn{3}{|c|}{ Treatments $^{1}$} & \multirow[b]{2}{*}{$P$-value } \\
\hline & CK & WF & GF & \\
\hline C6:0, caproic & $0.61 \pm 0.25$ & $0.50 \pm 0.23$ & $0.51 \pm 0.29$ & 0.597 \\
\hline C8:0, caprylic & $0.48 \pm 0.31$ & $0.50 \pm 0.30$ & $0.71 \pm 0.33$ & 0.230 \\
\hline C10:0, capric & $3.80 \pm 1.18$ & $2.24 \pm 1.62$ & $2.08 \pm 0.90$ & 0.606 \\
\hline C12:0, lauric & $2.16 \pm 0.94$ & $3.25 \pm 1.04$ & $3.12 \pm 1.17$ & 0.963 \\
\hline C14:0, myristic & $11.07 \pm 2.55$ & $11.91 \pm 2.16$ & $9.41 \pm 2.90$ & 0.192 \\
\hline C16:0, palmitic & $55.87 \pm 5.09^{b}$ & $57.72 \pm 2.74^{b}$ & $62.18 \pm 5.05^{a}$ & 0.013 \\
\hline C18:0, stearic & $6.69 \pm 0.83$ & $8.38 \pm 2.53$ & $7.16 \pm 1.39$ & 0.101 \\
\hline Other-C18: $1^{2}$ & $0.18 \pm 0.03$ & $0.22 \pm 0.05$ & $0.25 \pm 0.07$ & 0.057 \\
\hline t9-C18:1, elaidic acid & $0.04 \pm 0.01^{a}$ & $0.03 \pm 0.01^{b}$ & $0.04 \pm 0.01^{\mathrm{a}}$ & 0.018 \\
\hline c9,c12-C18:2, linoleic acid & $1.07 \pm 0.24$ & $0.86 \pm 0.32$ & $1.22 \pm 0.58$ & 0.164 \\
\hline c9,c12,c15-C18:3, (ALA) & $0.21 \pm 0.06^{b}$ & $0.44 \pm 0.26^{a}$ & $0.33 \pm 0.19^{a b}$ & 0.039 \\
\hline c5,c8,c11,c14c17-C20:5 (EPA) & $0.47 \pm 0.52$ & $0.85 \pm 0.69$ & $0.32 \pm 0.38$ & 0.099 \\
\hline Total n-3 PUFA & $0.67 \pm 0.50^{\mathrm{b}}$ & $1.25 \pm 0.67^{a}$ & $0.69 \pm 0.33^{b}$ & 0.036 \\
\hline C22:0, docosanoic acid & $0.03 \pm 0.01$ & $0.02 \pm 0.01$ & $0.02 \pm 0.01$ & 0.212 \\
\hline c3-C22:1, cis-3-Docosenoate & $0.08 \pm 0.02$ & $0.06 \pm 0.03$ & $0.07 \pm 0.04$ & 0.334 \\
\hline
\end{tabular}

ALA, $\alpha$-linolenic acid; EPA, eicosapentaenoic acid; $n$-3 PUFA, omega-3 polyunsaturated fatty acid.

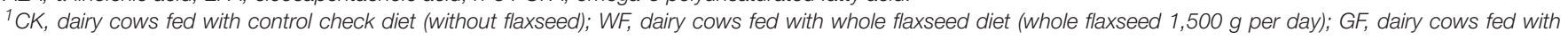
ground flaxseed diet (ground flaxseed 1,500 g per day).

${ }^{2}$ Other-C18:1 = c8-C18:1, c6-C18:1, and c9-C18:1.

a,b Means in the same row with different superscripts differ significantly in terms of treatment effect.

TABLE 3 | Effects of dietary whole and ground flaxseed supplementation on rumen fermentation indexes.

\begin{tabular}{|c|c|c|c|c|}
\hline \multirow[t]{2}{*}{ Items } & \multicolumn{3}{|c|}{ Treatments $^{1}$} & \multirow[b]{2}{*}{$P$-value } \\
\hline & CK & WF & GF & \\
\hline $\mathrm{pH}$ & $7.07 \pm 0.14$ & $6.94 \pm 0.23$ & $6.86 \pm 0.26$ & 0.115 \\
\hline $\mathrm{NH}_{3}-\mathrm{N}(\mathrm{mg} / \mathrm{dL})$ & $5.74 \pm 0.96$ & $6.53 \pm 1.93$ & $6.27 \pm 2.39$ & 0.630 \\
\hline \multicolumn{5}{|l|}{ Molar proportion, \% } \\
\hline Acetate & $60.25 \pm 1.58^{\mathrm{a}}$ & $52.02 \pm 1.85^{\mathrm{b}}$ & $48.29 \pm 2.61^{c}$ & $<0.001$ \\
\hline Propionate & $19.70 \pm 1.7^{\mathrm{b}}$ & $20.38 \pm 1.32^{a b}$ & $21.88 \pm 2.13^{\mathrm{a}}$ & 0.028 \\
\hline Isobutyrate & $1.81 \pm 0.23^{\mathrm{C}}$ & $3.05 \pm 0.30^{b}$ & $3.71 \pm 0.26^{c}$ & $<0.001$ \\
\hline Butyrate & $13.82 \pm 1.33^{c}$ & $17.30 \pm 1.13^{\mathrm{b}}$ & $20.53 \pm 1.04^{a}$ & $<0.001$ \\
\hline Isovalerate & $2.88 \pm 0.30^{c}$ & $3.64 \pm 0.42^{b}$ & $4.73 \pm 0.44^{\mathrm{a}}$ & $<0.001$ \\
\hline Valerate & $1.54 \pm 0.17^{c}$ & $2.10 \pm 0.14^{b}$ & $2.38 \pm 0.11^{\mathrm{a}}$ & $<0.001$ \\
\hline Acetate:propionate ratio & $3.08 \pm 0.31^{a}$ & $2.39 \pm 0.19^{b}$ & $2.40 \pm 0.32^{b}$ & $<0.001$ \\
\hline
\end{tabular}

$\mathrm{NH}_{3}-\mathrm{N}=$ ammonia- $\mathrm{N}$.

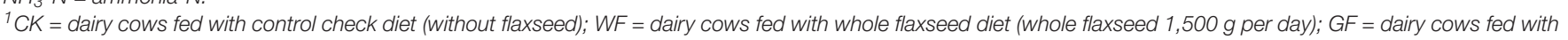
ground flaxseed diet (ground flaxseed 1,500 g per day).

${ }^{a-c}$ Means in the same row with different superscripts differ significantly in terms of treatment effect.

were lower in the GF group. The goods coverage value was highest in the GF group $(P=0.002)$ and was higher than 0.99 in all groups.

\section{Bacterial Composition}

In the rumen, bacteria are the main microbe and are divided into many different phylum or genus. At the phylum level, 19 taxa were detected in the rumen. Figure $1 \mathrm{~A}$ shows that the Bacteroidetes (CK: 64.77\%, WF: 56.53\%, GF: 42.84\%), Firmicutes (CK: 26.69\%; WF: 37.28\%; GF: 47.31\%), and Spirochaetes (CK: $2.90 \%$; WF: $1.37 \%$; GF: $0.49 \%$ ) were the main phyla in all groups. Flaxseed supplementation reduced the abundance of Bacteroidetes $(P<0.001)$ and Spirochaetes $(P<0.001)$, whereas Firmicutes improved $(P<0.001)$ in rumen. At the genus level, a total of 354 genera were detected in the rumen. As shown in Figure 1B and Table 5, the unclassified sequences in CK, WF, and GF were $27.49 \%, 19.94 \%$, and 19.29\%, respectively, and Prevotella_1 (CK 30.41\%; WF 29.76\%; GF $23.19 \%$ ) was the most abundant genera in rumen. The results of this experiment showed that feeding a GF diet could reduce the abundance of Rikenellaceae_RC9_gut_group $(P=0.042)$ compared with the CK and WF diet. The abundance of Succiniclasticum ( $P=0.005)$, Prevotellaceae_UCG-001 $(P<0.001)$, Treponema $2(P<0.001)$, and Fibrobacter 
TABLE 4 | Effects of dietary whole and ground flaxseed supplementation on $\alpha$-diversity indexes of rumen bacteria.

\begin{tabular}{|c|c|c|c|c|}
\hline \multirow[t]{2}{*}{ Items } & \multicolumn{3}{|c|}{ Treatments $^{1}$} & \multirow[t]{2}{*}{$P$-value } \\
\hline & CK & WF & GF & \\
\hline Ace & $1,960.48 \pm 188.81^{a b}$ & $2,006.18 \pm 101.02^{a}$ & $1,820.80 \pm 173.69^{b}$ & 0.039 \\
\hline Chao & $1,918.23 \pm 167.97$ & $1,973.29 \pm 90.48$ & $1,833.69 \pm 166.70$ & 0.118 \\
\hline Goods_coverage & $0.9939 \pm 0.0017^{b}$ & $0.9933 \pm 0.0015^{b}$ & $0.9958 \pm 0.0013^{a}$ & 0.002 \\
\hline Shannon & $8.59 \pm 0.09^{a}$ & $8.42 \pm 0.15^{a}$ & $7.90 \pm 0.46^{b}$ & $<0.001$ \\
\hline Simpson & $0.9934 \pm 0.0013^{a}$ & $0.9929 \pm 0.0011^{a}$ & $0.9829 \pm 0.0121^{b}$ & 0.004 \\
\hline Sobs & $1,687.30 \pm 165.16$ & $1,690.70 \pm 88.42$ & $1,554.60 \pm 190.48$ & 0.098 \\
\hline
\end{tabular}

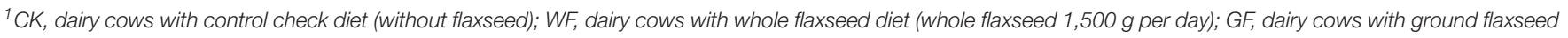
diet (ground flaxseed 1,500 g per day).

${ }^{a, b}$ Means in the same row with different superscripts differ significantly in terms of treatment effect.

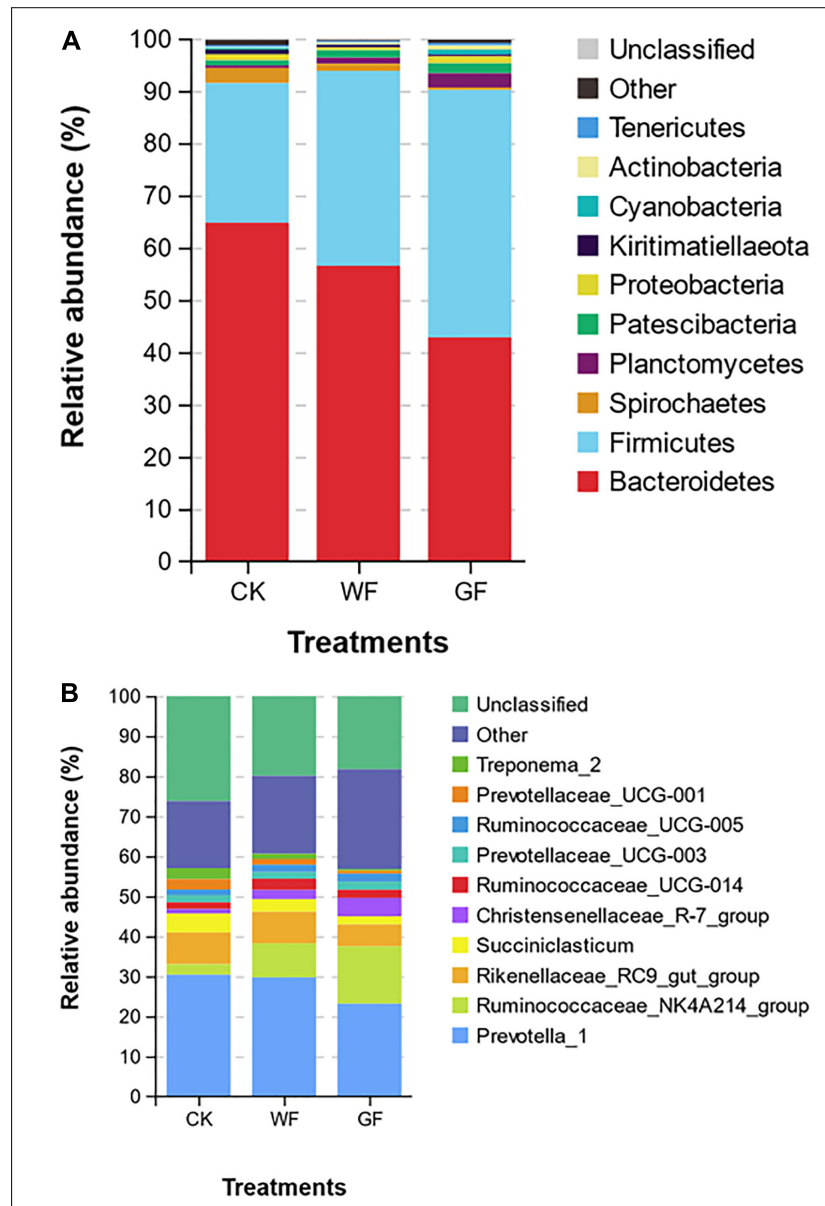

FIGURE 1 | (A) Relative abundance of the bacterial community on the phylum level (only the top 10 abundant phyla are presented). (B) Relative abundance of bacterial communities on the genus level (only the top 10 abundant genera are presented). CK, dairy cows fed with control check diet (without flaxseed); WF, dairy cows fed with whole flaxseed diet (whole flaxseed 1,500 g per day); GF, dairy cows with ground flaxseed diet (ground flaxseed 1,500 g per day).

$(P<0.001)$ showed a similar trend: $\mathrm{CK}>\mathrm{WF}>\mathrm{GF}$. However, the abundance of Ruminococcaceae_NK4A214_group $(P<0.001)$, Christensenellaceae_R-7_group $(P=0.001)$,
Eubacterium coprostanoligenes $(P=0.005)$, Candidatus saccharimonas $(P=0.002)$, the $p$-1088-a5 gut group $(P<0.001)$, and Butyrivibrio_2 $(P=0.047)$ showed a CK $<$ WF $<$ GF trend.

\section{Principal Coordinate Analysis}

We used PCoA to compare the OTU among the CK, WF, and GF groups $(\mathrm{PCo} 1=29.98 \%$; PCo2 $=15.28 \%)$. As shown in Figure 2A, a clear separation was observed in the plot of the CK and GF groups and could be well distinguished with the value of PCo1. On the PCol axis, most GF was distributed in the positive score values, whereas most $\mathrm{CK}$ was in the negative. However, it was difficult to distinguish between the CK and GF groups with the value of PCo2. At the same time, WF could not be separated from the CK and GF groups. Comparisons of ANOSIM test showed significant differences in genus level between $\mathrm{CK}$, WF, and GF $(R=0.598 ; P=0.001)$ (as shown in Figure 2B). This result revealed that a change in diet composition influenced the composition of the microorganisms in the rumen.

\section{DISCUSSION}

The ALA in WF and that in GF were released in different ways. The GF could release the ALA into the rumen directly, and approximately $4 \mathrm{~h}$ later, the PUFA concentration was back to normal (Baldin et al., 2018). In this experiment, rumen fluid was collected more than $4 \mathrm{~h}$ after feeding; thus, no difference was shown between the concentrations of ALA, EPA, and total n-3 PUFA in the CK and GF groups. However, because of the shell, the ALA in WF could only be released after rumination, and this release may last longer. Thus, higher concentrations of ALA, EPA, and total n-3 PUFA were found in the WF group, but not all WF can be broken or crushed through rumination, and therefore, many were excreted in the stools (Oba et al., 2009). Thus, higher concentrations of ALA, EPA, and n-3 PUFA in milk were found in the GF group, compared to the WF group.

In this study, flaxseed supplementation had no effect on rumen $\mathrm{pH}$ and $\mathrm{NH}_{3}-\mathrm{N}$. Similarly, a previous study also reported that flaxseed oil supplementation had no effect on the $\mathrm{pH}$ and $\mathrm{NH}_{3}-\mathrm{N}$ in the rumen (Pi et al., 2019). However, supplementation with ALA can modify the molar proportion of VFAs in the rumen (Gao et al., 2016). VFAs are important metabolites that 
TABLE 5 | Effects of dietary whole and ground flaxseed supplementation on rumen bacterial genera ${ }^{1}$.

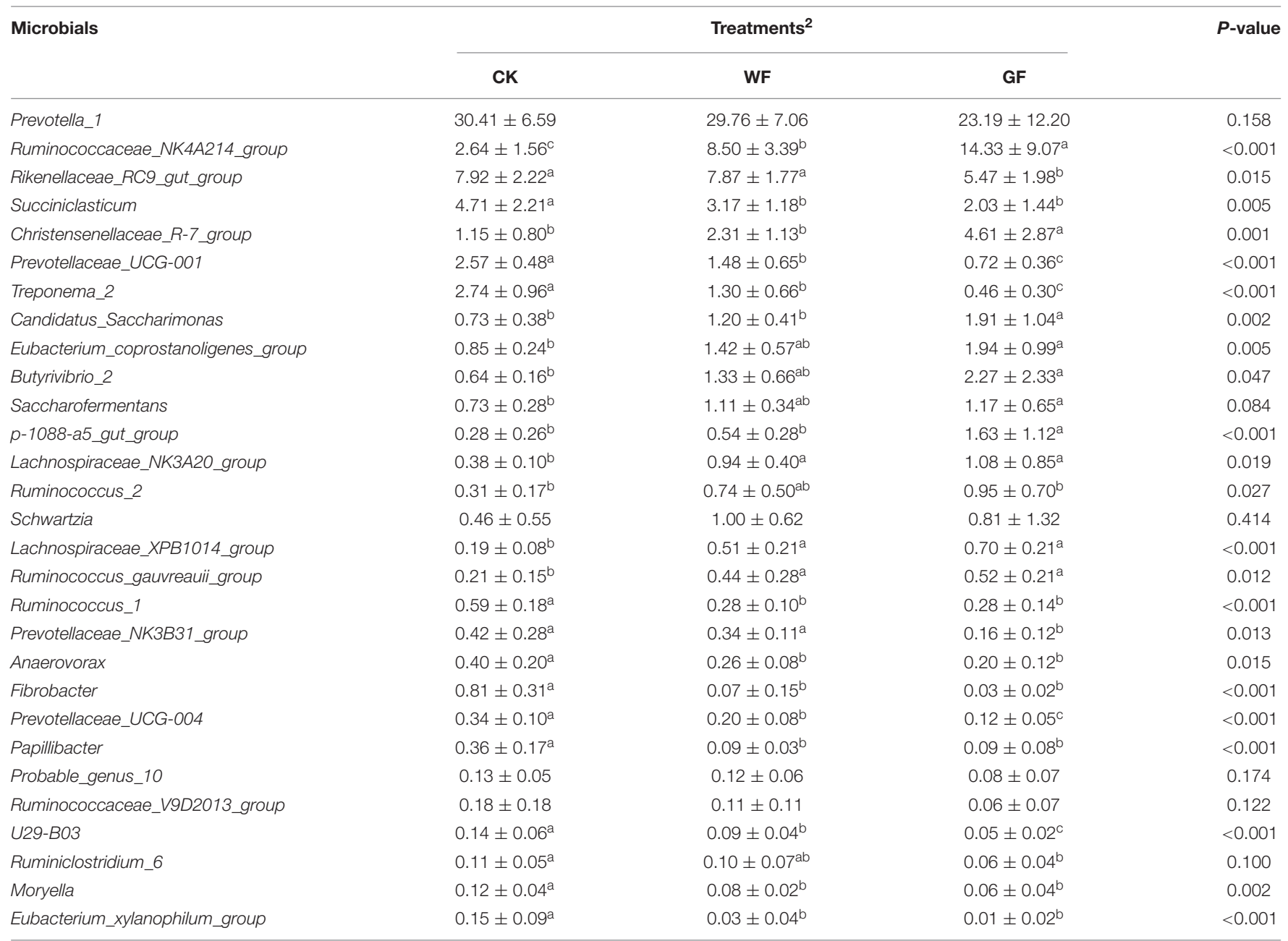

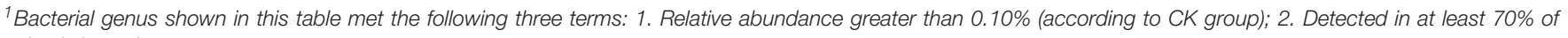
animals in each group.

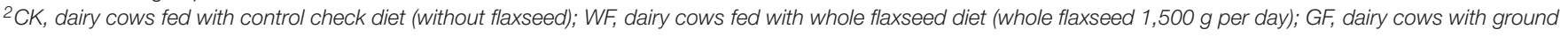
flaxseed diet (ground flaxseed 1,500 g per day).

${ }^{a-c}$ Means in the same row with different superscripts differ significantly in terms of treatment effect.

are produced by rumen fermentation and can provide $70 \%$ of the digestible energy required by the host (Bergman, 1990). Previous studies have reported that GF supplementation reduced the molar proportion of acetate and increased propionate in the rumen (Velez, 2012; Isenberg, 2014). The same result was also reported with WF supplementation in diet (Gutierrezgomez et al., 2020). However, a study by Kholif et al. (2018) found that GF supplementation in the diet enhanced the molar proportion of propionate but had no effect on acetate in the rumen. This might be attributed to the differences in animal species and flaxseed form. Kholif et al. (2018) chose goats and crushed flaxseed for their experiment, whereas this study used dairy cows and GF. The flaxseed form can influence the release of oil from seeds (Kholif et al., 2018). GF supplementation showed a greater influence on the molar proportion of VFAs in the rumen compared with WF supplementation. ALA in crushed flaxseed may release more slowly during digestion and fermentation and showed no effect on the molar proportion of acetate in the rumen. In this study, GF supplementation showed greater influence on the molar proportion of VFAs in the rumen compared to WF supplementation. Feeding flaxseed reduced the ratio of acetate to propionate, and this result was the same in a study by Castagnino et al. (2015). Flaxseed supplementation can shift rumen fermentation to propionate at the expense of acetate (Vargas et al., 2020). But no difference was shown in the ratio of acetate to propionate. In ruminants, rumen microbes play an essential role in shaping the VFAs in the rumen (Mcallister et al., 1994; Xue et al., 2019).

Diet is the main factor in shaping the bacterial communities in the rumen (Ley et al., 2008; Henderson et al., 2015). The addition of fatty acids, especially PUFA, could influence the microbial community (Enjalbert et al., 2017). GF supplementation could change the abundance of dominant phylum in the rumen compared to the CK and WF groups. Bacteroidetes species have a 


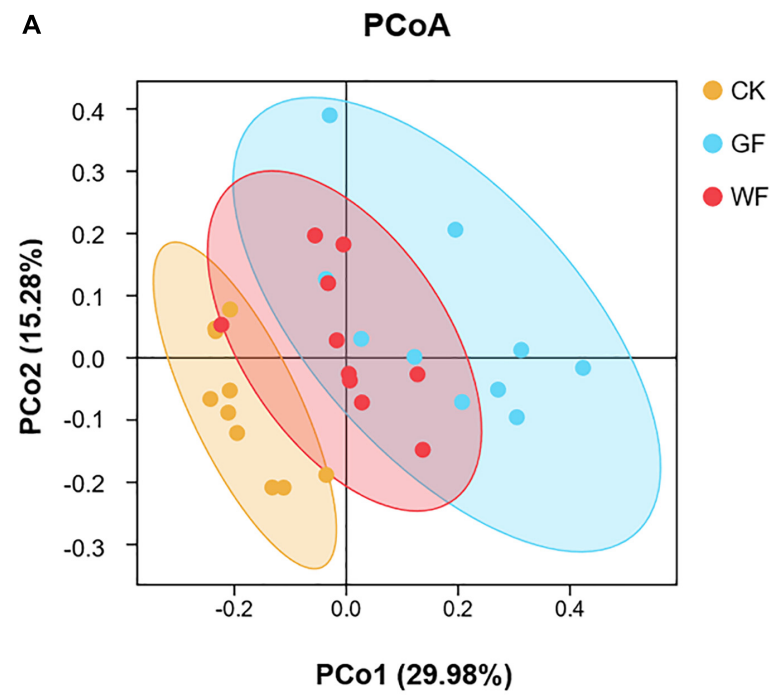

B

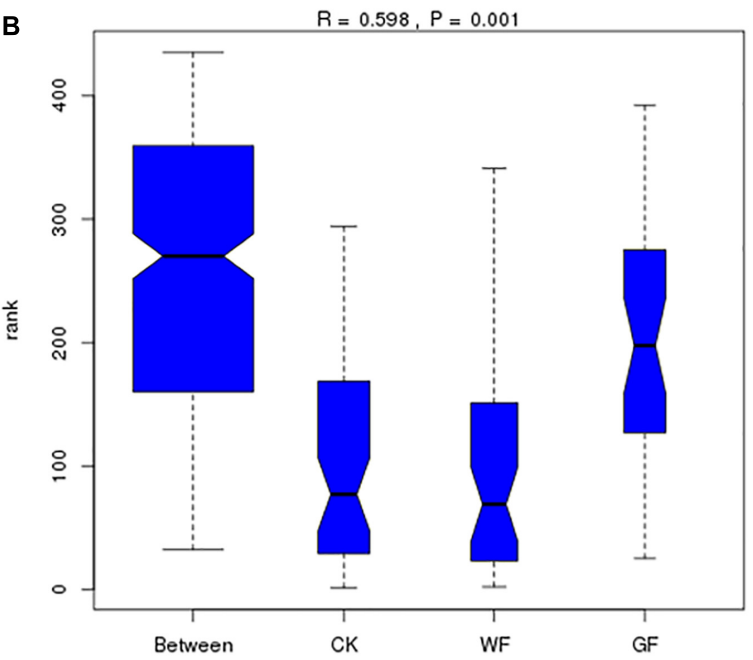

FIGURE 2 | (A) Principal coordinate analysis of rumen bacterial samples; (B) similarity of bacterial genera among the three groups. CK, dairy cows fed with control check diet (without flaxseed); WF, dairy cows fed with whole flaxseed diet (whole flaxseed 1,500 g per day); GF, dairy cows fed with ground flaxseed diet (ground flaxseed 1,500 g per day).

high correlation with enzymes involved in acetate and butyrate, and Firmicutes species are positive with ruminal butyrate (Deusch et al., 2017). Indeed, this experiment also observed that the lowest molar proportion of acetate and highest abundance of Bacteroidetes and the highest molar proportion of butyrate and the lowest abundance of Firmicutes were all found in the GF group. At the genus level, the relative abundances of bacteria Treponema 2, Prevotellaceae_UCG-001, and Succiniclasticum were significantly lower in the GF and WF groups than in the CK group, and lower in the GF group than in the WF group. The results also showed that Treponema_2 and Prevotellaceae UCG001 contributed to higher acetate and propionate concentrations. Treponema species, a common bacterial group, can digest soluble fibers (Bekele et al., 2011); Treponema 2 belongs to this group. Prevotellaceae_UCG-001 belongs to the Prevotellaceae family, which has a positive association with digestive proteins and amino acids (Zhao et al., 2017; Zhu et al., 2019), and is unable to degrade fibers. However, Prevotellaceae has the ability to promote fiber degradation, when cocultured with cellulolytic bacteria (Morotomi et al., 2009; Rosenberg, 2014). Other studies have reported that Succiniclasticum was involved mainly in the fermentation of succinate to propionate (Gylswyk, 1995). In this study, our results also showed a correlation between Succiniclasticum and the molar proportion of propionate.

Previous research a high correlation between Ruminococcaceae_NK4A214_group and rumen biohydrogenation of $9 \mathrm{c}-\mathrm{C} 18: 1$ to $\mathrm{C} 18: 0$ (Wang X. et al., 2019). Ruminococcaceae_NK4A214_group. Ruminococcaceae_NK4A214_group and Eubacterium_ coprostanoligenes_group all belong to Ruminococcaceae. In addition, Ruminococcaceae may play a predominant role in biohydrogenation in the rumen (Huws et al., 2011). In this study, the GF group had a higher abundance of Ruminococcaceae_NK4A214_group compared to the WF group. This can be associated with more ALA released in the rumen from GF supplementation. Christensenellaceae_R-7_group showed the same trend as Ruminococcaceae_NK4A214_group and Eubacterium coprostanoligenes between the treatments in this experiment. There are no studies that report a relationship between the Christensenellaceae_R-7_group and Eubacterium coprostanoligenes in rumen biohydrogenation, but many studies have found a positive correlation between the Ruminococcaceae_NK4A214_group, Christensenellaceae_R7_group, and Eubacterium coprostanoligenes with milk yield (Tong et al., 2018; Sun et al., 2019). Although the three bacteria do not all belong to the same family (Ruminococcaceae: Ruminococcaceae_NK4A214_group and Christensenellaceae_R7_group; Christensenellaceae: Eubacterium coprostanoligenes), they might belong to the same functional group (Morais and Mizrahi, 2019). The Christensenellaceae_R-7_group and Eubacterium_coprostanoligenes_group may promote rumen biohydrogenation alone or cooperate with others.

\section{CONCLUSION}

Different forms of flaxseed released ALA differently in the rumen. WF released the ALA over a long time, whereas GF released ALA over a shorter time, which had a different effect on the composition of fatty acids in the rumen. The composition of microorganisms and the molar proportion of VFA in the rumen showed the same trend with different flaxseed form supplementation. Supplementation with GF changed the dominant bacterial at the phylum level and increased the abundance of the Ruminococcaceae_NK4A214_group, Christensenellaceae_R7_group, and Eubacterium coprostanoligenes groups. In addition, the Ruminococcaceae_NK4A214_group contributed to rumen biohydrogenation, and the Christensenellaceae_R-7_group and Eubacterium coprostanoligenes groups may promote biohydrogenation. Further study using metagenomic and metatranscriptomic analyses of the microbial metabolic 

biohydrogenation is needed.

\section{DATA AVAILABILITY STATEMENT}

The datasets presented in this study can be found in online repositories. The names of the repository/repositories and accession number(s) can be found below: NCBI SRA; PRJNA756791.

\section{ETHICS STATEMENT}

The animal study was reviewed and approved by Animal Care and Use Committee of the Institute of Animal Science, Chinese Academy of Agricultural Sciences. Written informed consent was obtained from the owners for the participation of their animals in this study.

\section{REFERENCES}

Baldin, M., Rico, D. E., Green, M. H., and Harvatine, K. J. (2018). An in vivo method to determine kinetics of unsaturated fatty acid biohydrogenation in the rumen. J. Dairy Sci. 101, 4259-4267.

Bekele, A. Z., Koike, S., and Kobayashi, Y. (2011). Phylogenetic diversity and dietary association of rumen Treponema revealed using group-specific $16 \mathrm{~S}$ rRNA gene-based analysis. FEMS Microbiol. Lett. 316, 51-60. doi: 10.1111/j. 1574-6968.2010.02191.x

Bergman, E. N. (1990). Energy contributions of volatile fatty acids from the gastrointestinal tract in various species. Physiol. Rev. 70, 567-590. doi: 10.1152/ physrev.1990.70.2.567

Brzozowska, A. M., Micek, P., Kuczynska, B., Lukaszewicz, M., and Oprzadek, J. M. (2018). The use of energy-protein supplement increases performance of highyielding dairy cows and improves health-promoting properties of milk. Anim. Prod. Sci. 58, 1708-1713. doi: 10.1071/an16161

Castagnino, P. S., Messana, J. D., Fiorentini, G., De Jesus, R. B., Vito, E. S., Carvalho, I. P., et al. (2015). Glycerol combined with oils did not limit biohydrogenation of unsaturated fatty acid but reduced methane production in vitro. Anim. Feed Sci. Technol. 201, 14-24. doi: 10.1016/j.anifeedsci.2014.12.004

Castillo-Lopez, E., Moats, J., Aluthge, N. D., Ramirez Ramirez, H. A., Christensen, D. A., Mutsvangwa, T., et al. (2018). Effect of partially replacing a barley-based concentrate with flaxseed-based products on the rumen bacterial population of lactating Holstein dairy cows. J. Appl. Microbiol. 124, 42-57. doi: 10.1111/jam. 13630

Deusch, S., Camarinhasilva, A., Conrad, J., Beifuss, U., Rodehutscord, M., and Seifert, J. (2017). A structural and functional elucidation of the rumen microbiome influenced by various diets and microenvironments. Front. Microbiol. 8:1605. doi: 10.3389/fmicb.2017.01605

Dreiucker, J., and Vetter, W. (2011). Fatty acids patterns in camel, moose, cow and human milk as determined with GC/MS after silver ion solid phase extraction. Food Chem. 126, 762-771. doi: 10.1016/j.foodchem.2010.11.061

Enjalbert, F., Combes, S., Zened, A., and Meynadier, A. (2017). Rumen microbiota and dietary fat: a mutual shaping. J. Appl. Microbiol. 123, 782-797. doi: 10.1111/ jam.13501

Feng, Z. C., and Gao, M. (1993). The determination of ammonia nitrogen concentration in the rumen fluid by a colorimetric method. Inner Mong. J. Anim. Sci. 4, 40-41.

Fievez, V., Boeckaert, C., Vlaeminck, B., Mestdagh, J., and Demeyer, D. (2007). In vitro examination of dha-edible micro-algae:2. Effect on rumen methane production and apparent degradability of hay. Anim. Feed Sci. Technol. 136, 80-95. doi: 10.1016/j.anifeedsci.2006. 08.016

\section{AUTHOR CONTRIBUTIONS}

GH: conceptualization, software, data curation, and writing original draft. LG: methodology. XC: data curation. KL: software. WT: resources. SZ: formal analysis. YZ: writing - review and editing. NZ: supervision. JW: project administration. All authors contributed to the article and approved the submitted version.

\section{FUNDING}

This study was financially supported by the Agricultural Science and Technology Innovation Program (ASTIP-IAS12), the Modern Agro-Industry Technology Research System of the PR China (CARS-36), and the Scientific Research Project for Major Achievements of the Agricultural Science and Technology Innovation Program (CAAS-ZDXT2019004).

Gao, J., Wang, M., Jing, Y., Sun, X. Z., Tianyi, W. U., and Shi, L. (2016). Impacts of the unsaturation degree of long-chain fatty acids on the volatile fatty acid profiles of rumen microbial fermentation in goats in vitro. J. Integr. Agric. 15, 2827-2833.

Gutierrezgomez, C., Vera, N., Allende, R., Williams, P., Astudillo, R., and Avilastagno, J. (2020). Linseed and glycerol in forage diets effect methane production and rumen fermentation parameters in a Rusitec semi-continuos system. Anim. Prod. Sci. 60, 923-929. doi: 10.1071/an18710

Gylswyk, N. O. V. (1995). Succiniclasticum ruminis gen. nov. sp. nov. a ruminal bacterium converting succinate to propionate as the sole energy-yielding mechanism. Int. J. Syst. Bacteriol. 45, 297-300. doi: 10.1099/00207713-45-2297

Henderson, G., Cox, F., Ganesh, S., Jonker, A., Young, W., and Janssen, P. H. (2015). Rumen microbial community composition varies with diet and host, but a core microbiome is found across a wide geographical range. Sci. Rep. 5:14567.

Hu, W. L., Liu, J. X., Ye, J. A., Wu, Y. M., and Guo, Y. Q. (2005). Effect of tea saponin on rumen fermentation in vitro. Anim. Feed Sci. Technol. 120, 333-339. doi: 10.1016/j.anifeedsci.2005.02.029

Huws, S. A., Kim, E. J., Lee, M. R. F., Scott, M. B., Tweed, J. K. S., Pinloche, E., et al. (2011). As yet uncultured bacteria phylogenetically classified as Prevotella, Lachnospiraceae incertae sedis and unclassified Bacteroidales, Clostridiales and Ruminococcaceae may play a predominant role in ruminal biohydrogenation. Environ. Microbiol. 13, 1500-1512. doi: 10.1111/j.1462-2920.2011.02452.x

Isenberg, B. J. (2014). Effects of Ground Flaxseed Supplementation on Animal Production and Milk Fatty Acid Profile in Organically-Certified Lactating Jerseys During the Grazing Season. Ph.D. thesis. Durham, NH: University of New Hampshire.

Isenberg, B. J., Soder, K. J., Pereira, A. B., Standish, R., and Brito, A. F. (2019). Production, milk fatty acid profile, and nutrient utilization in grazing dairy cows supplemented with ground flaxseed. J. Dairy Sci. 102, 1294-1311. doi: 10.3168/jds.2018-15376

Kholif, A. E., Morsy, T. A., and Abdo, M. M. (2018). Crushed flaxseed versus flaxseed oil in the diets of Nubian goats: effect on feed intake, digestion, ruminal fermentation, blood chemistry, milk production, milk composition and milk fatty acid profile. Anim. Feed Sci. Technol. 244, 66-75.

Ley, R. E., Hamady, M., Lozupone, C. A., Turnbaugh, P. J., Ramey, R. R., Bircher, J. S., et al. (2008). Evolution of mammals and their gut microbes. Science 320, 1647-1651.

Maia, M., Chaudhary, L. C., Figueres, L., and Wallace, R. J. (2007). Metabolism of polyunsaturated fatty acids and their toxicity to the microflora of the rumen. Antonie Van Leeuwenhoek 91, 303-314. doi: 10.1007/s10482-006-9118-2

Marino, R., Malva, A. D., Caroprese, M., Palo, P. D., Santillo, A., Sevi, A., et al. (2019). Effects of whole linseed supplementation and treatment duration on 
fatty acid profile and endogenous bioactive compounds of beef muscle. Animal 13, 444-452. doi: 10.1017/S1751731118001635

Mcallister, T. A., Bae, H. D., Jones, G. A., and Cheng, K. J. (1994). Microbial attachment and feed digestion in the rumen. J. Anim. Sci. 72, 3004-3018. doi: $10.2527 / 1994.72113004 x$

Meignan, T., Lechartier, C., Chesneau, G., and Bareille, N. (2017). Effects of feeding extruded linseed on production performance and milk fatty acid profile in dairy cows: a meta-analysis. J. Dairy Sci. 100, 4394-4408. doi: 10.3168/jds.201611850

Mesgaran, M. D., Jafarpoor, R. J., and Mesgaran, S. D. (2012). Milk production, milk fatty acid composition, and blood biochemical parameters of holstein dairy cows fed whole or ground flaxseed instead of extruded soybeans in the first half of lactation. Iran. J. Vet. Res. 13, 203-209.

Morais, S., and Mizrahi, I. (2019). The road not taken: the rumen microbiome, functional groups, and community states. Trends Microbiol. 27, 538-549. doi: 10.1016/j.tim.2018.12.011

Morotomi, M., Nagai, F., Sakon, H., and Tanaka, R. (2009). Paraprevotella clara gen. nov., sp. nov. and Paraprevotella xylaniphila sp. nov., members of the family 'Prevotellaceae' isolated from human faeces. Int. J. Syst. Evol. Microbiol. 59, 1895-1900. doi: 10.1099/ijs.0.008169-0

Mustafa, A. F., Mckinnon, J. J., Christensen, D. A., and He, T. (2002). Effects of micronization of flaxseed on nutrient disappearance in the gastrointestinal tract of steers. Anim. Feed Sci. Technol. 95, 123-132. doi: 10.1016/s0377-8401(01) 00337-6

Oba, M., Thangavelu, G., Dehghan-Banadaky, M., and Ambrose, D. J. (2009). Unprocessed whole flaxseed is as effective as dry-rolled flaxseed at increasing $\alpha$-linolenic acid concentration in milk of dairy cows. Livest. Sci. 122, 73-76. doi: 10.1016/j.livsci.2008.07.012

Paz, H. A., Anderson, C. L., Muller, M. J., Kononoff, P. J., and Fernando, S. C. (2016). Rumen bacterial community composition in Holstein and Jersey cows is different under same dietary condition and is not affected by sampling method. Front. Microbiol. 7:1206. doi: 10.3389/fmicb.2016. 01206

Petit, H. V. (2003). Digestion, milk production, milk composition, and blood composition of dairy cows fed formaldehyde treated flaxseed or sunflower seed. J. Dairy Sci. 86, 2637-2646.

Petit, H. V., and Cortes, C. (2010). Milk production and composition, milk fatty acid profile, and blood composition of dairy cows fed whole or ground flaxseed in the first half of lactation. Anim. Feed Sci. Technol. 158, 36-43. doi: 10.1016/j. anifeedsci.2010.03.013

Pi, Y., Ma, L., Pierce, K. M., Wang, H. R., Xu, J. C., and Bu, D. P. (2019). Rubber seed oil and flaxseed oil supplementation alter digestion, ruminal fermentation and rumen fatty acid profile of dairy cows. Animal 13, 2811-2820. doi: 10.1017/ S175173111900137X

Rosenberg, E. (2014). “The family Prevotellaceae, in The Prokaryotes, eds E. Rosenberg, E.F. DeLong, S. Lory, E. Stackebrandt, and F. Thompson (Berlin: Springer), 825-827. doi: 10.1007/978-3-642-38954-2_131

Silva, D., Santos, G. T., Branco, A. F., Damasceno, J. C., Kazama, R., Matsushita, M., et al. (2007). Production performance and milk composition of dairy cows fed whole or ground flaxseed with or without monensin. J. Dairy Sci. 90, 2928-2936. doi: $10.3168 /$ jds.2006-573
Sun, Z., Yu, Z., and Wang, B. (2019). Perilla frutescens leaf alters the rumen microbial community of lactating dairy cows. Microorganisms 7:562. doi: 10 . 3390/microorganisms7110562

Tong, J., Zhang, H., Yang, D., Zhang, Y., Xiong, B., and Jiang, L. (2018). Illumina sequencing analysis of the ruminal microbiota in high-yield and lowyield lactating dairy cows. PLoS One 13:e0198225. doi: 10.1371/journal.pone. 0198225

Vargas, J. E., Andrés, S., López-Ferreras, L., Snelling, T. J., Yáñez-Ruíz, D. R., García-Estrada, C., et al. (2020). Dietary supplemental plant oils reduce methanogenesis from anaerobic microbial fermentation in the rumen. Sci. Rep. 10:1613. doi: 10.1038/s41598-020-58401-z

Velez, K. (2012). Effects of Incremental Dietary Levels of Ground Flaxseed on Milk Production, Ruminal Metabolism, and Enteric Methane Emissions in Organic Dairy Cows. Honor Theses and Capstones, 26.

Wang, C., He, L. W., Xing, Y. Q., Zhou, W., Yang, F. Y., Chen, X. Y., et al. (2019). Fermentation quality and microbial community of alfalfa and stylo silage mixed with Moringa oleifera leaves. Bioresour. Technol. 284, 240-247. doi: 10.1016/j.biortech.2019.03.129

Wang, X., Martin, G. B., Wen, Q., Liu, S., Zhang, J., Yu, Y., et al. (2019). Linseed oil and heated linseed grain supplements have different effects on rumen bacterial community structures and fatty acid profiles in cashmere kids. J. Anim. Sci. 97, 2099-2113. doi: 10.1093/jas/skz079

Xue, M. Y., Sun, H. Z., Wu, X. H., Guan, L. L., and Liu, J. X. (2019). Assessment of rumen bacteria in dairy cows with varied milk protein yield. J. Dairy Sci. 102, 5031-5041. doi: 10.3168/jds.2018-15974

Zhao, X. H., Chen, Z. D., Zhou, S., Song, X. Z., Ouyang, K. H., Pan, K., et al. (2017). Effects of daidzein on performance, serum metabolites, nutrient digestibility, and fecal bacterial community in bull calves. Anim. Feed Sci. Technol. 225, 87-96. doi: 10.1016/j.anifeedsci.2017.01.014

Zhu, H. Z., Liang, Y. D., Ma, Q. Y., Hao, W. Z., Li, X. J., Wu, M. S., et al. (2019). Xiaoyaosan improves depressive-like behavior in rats with chronic immobilization stress through modulation of the gut microbiota. Biomed. Pharmacother. 112:108621. doi: 10.1016/j.biopha.2019.108621

Conflict of Interest: The authors declare that the research was conducted in the absence of any commercial or financial relationships that could be construed as a potential conflict of interest.

Publisher's Note: All claims expressed in this article are solely those of the authors and do not necessarily represent those of their affiliated organizations, or those of the publisher, the editors and the reviewers. Any product that may be evaluated in this article, or claim that may be made by its manufacturer, is not guaranteed or endorsed by the publisher.

Copyright (c) 2021 Huang, Guo, Chang, Liu, Tang, Zheng, Zhao, Zhang and Wang. This is an open-access article distributed under the terms of the Creative Commons Attribution License (CC BY). The use, distribution or reproduction in other forums is permitted, provided the original author(s) and the copyright owner(s) are credited and that the original publication in this journal is cited, in accordance with accepted academic practice. No use, distribution or reproduction is permitted which does not comply with these terms. 